\title{
载人航天工程巡天空间望远镜大视场多色成像 与无缝光谱巡天
}

詹虎 ${ }^{1,2}$

1. 中国科学院国家天文台, 北京 100101 ;

2. 北京大学科维理天文与天体物理研究所, 北京 100871

E-mail: zhanhu@nao.cas.cn

近20年来, 以Sloan Digital Sky Survey(SDSS ${ }^{[1]}$ 为代表的 巡天项目取得了令人瞩目的成果，打开了用天文观测精确检 验和探索基础物理理论的新篇章, 催生了Vera Rubin Observatory(VRO, 原名Large Synoptic Survey Telescope)、Dark Energy Spectroscopic Instrument(DESI)、Euclid、Roman Space Telescope(RST, 原名Wide Field Infrared Survey Telescope)等新一 代大型巡天项目．在此背景下，载人航天工程巡天空间望远 镜(CSST, 图1; 巡天空间望远镜曾被称为中国空间站望远镜 (China Space Station Telescope, CSST), 目前沿用CSST的简 称)也应运而生, 预计 2024 年前后投人科学运行, 将主要用于 开展大规模多色成像与无缝光谱巡天工作，并可采用多种观 测手段对遴选的天体或天区进行更为深人的研究 ${ }^{[2 \sim 4]}$. 这些 国际天文界的标志性项目均以暗能量、暗物质、引力理论 等方向的研究为主要牵引, 同时也将极大地推进从太阳系到 宇宙学的各个方向的研究.

我国载人航天工程致力将载人空间站建设成为一个支 撑空间科学与应用前沿领域研究的国家级实验平台. 为此, 中国科学院空间科学与应用总体部(即载人航天工程空间应 用系统的总部，以下简称“总体部”) 2009 年12月组织召开一 系列研讨会, 探讨空间站在微重力科学、天文学、生命科

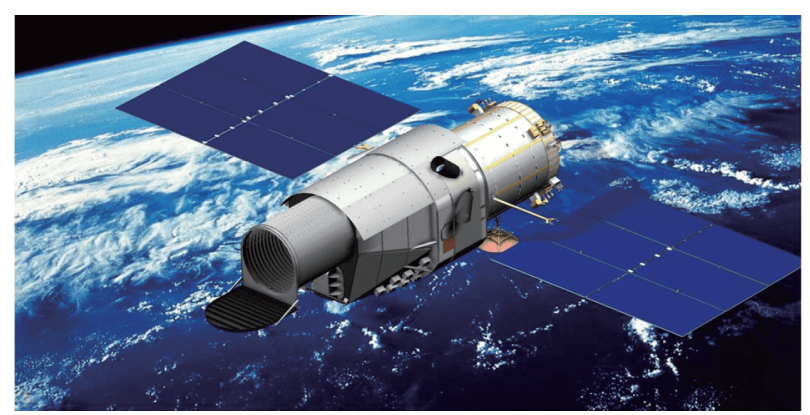

图 1 CSST在轨运行效果图(中国科学院长春光学精密机械与物理 研究所提供)

Figure 1 An artist rendition of CSST in orbit (provided by Changchun Institute of Optics, Fine Mechanics and Physics, Chinese Academy of Sciences)

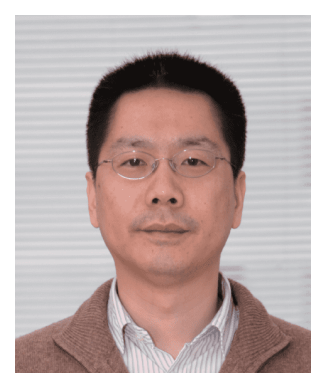

\begin{abstract}
詹虎中国科学院国家天文台研究 员、北京大学科维理天文与天体物 理研究所兼职教授, 巡天空间望远镜 科学工作委员会成员、一体化设计 总体技术组成员、巡天光学设施责 任科学家、巡天模块责任科学家与 主任设计师. 主要研究暗能量探测手 段和光学天文相机技术.
\end{abstract}

学、地球科学等领域的科学目标与研究方向, 从此拉开了 CSST项目的序幕. 经过两年的科学目标论证和技术方案论 证, 在空间站实验舱II上装载一架 $2 \mathrm{~m}$ 口径的光学望远镜进行 巡天观测和太赫兹谱线巡测的计划得到了广泛的认可, 并于 2012年2月底至3月初，经空间站任务规划委员会评议推荐, 获得工程总体的优先支持. 翌年4月, CSST完成了多方案择 优, 于11月正式立项.

在当时的方案中, CSST与实验舱II直接相连, 空间站组 合体的姿态变化、结构形变以及各种振源对其形成的扰动 会使凝视观测的像质严重退化, 因此需要采取一系列主动和 被动的措施消减扰动对观测的影响. 这些措施的技术难度较 大, 存在一定的风险. 此外, 空间站周围可能存在的污染环境 和颗粒物、空间站大致对地定向的姿态(对天体凝视观测期 间，需补偿约 $4 \% \mathrm{~min}$ 的空间站姿态变化)、其结构对观测方向 的限制以及舱体和太阳帆板等各处表面产生的杂散光等因 素, 都不利于天文观测. 为了改善CSST观测运行的条件, 中国 科学院和航天五院多位专家先后提议将其改为独立的空间 望远镜 ${ }^{[5]}$ ，并于2014年初启动了论证工作. 经过一年多的论 证, 工程总体批准了CSST与空间站共轨独立飞行的方案. 基 于此方案, CSST在常规运行期间与空间站保持一定的轨道相 位差, 定期或根据需要与空间站对接, 补给燃料和进行维 护、维修与升级.

为了进一步提升CSST的科学效益, 在工程总体的支持 下，总体部于 2018 年4月启动了新模块的论证, 其中三项建议 
通过遴选, 获得批准立项. 至此, CSST配备了 5台第一代仪器: 巡天模块、太赫兹模块、多通道成像仪、积分视场光谱仪 和系外行星成像星冕仪，巡天模块将以接近哈勃空间望远镜 (Hubble Space Telescope, HST)的角分辨率, 获取约17500平方 度天区的多色成像数据、同样天区的无㖓光谱数据和深场 观测数据, 波长覆盖255 1000 nm(部分视场0.9 1.7 $\mu \mathrm{m}$ ). 太赫 兹模块工作于 $0.41 \sim 0.51 \mathrm{THz}$ 频段 (590 730 $\mu \mathrm{m})$, 将开展银河 系内及近邻星系的分子谱线巡测. 多通道成像仪对约 50 平方 角分的视场分光三色, 实现 3 个滤光片的同视场同时观测. 积 分视场光谱仪对二维图像中的每一个空间分辨单元都可采 集光谱信息, 是研究近邻星系和黑洞周围环境及其物理过程 的利器. 星冕仪将在可见光波段实现对系外行星 $10^{-8}$ 以上的 高对比度直接成像观测研究.

在科学准备工作方面, CSST论证初期对科学数据处理 (子)系统作了初步的规划, 该系统于 2020 年底完成了立项程 序，研制工作已经全面展开. 与此同时，CSST科学工作委员 会根据广泛征集的研究课题, 梳理和规划了宇宙学、星系与 活动星系核、银河系与近邻星系、恒星科学、系外行星与 太阳系天体、天体测量和暂现源与变源7个研究方向共 24 个 课题. 这些课题涵盖了CSST高级数据处理、模拟仿真、采用 模拟数据和现有望远镜观测数据的研究、研究方向的深化 论证与规划、数据分析方法与算法研究、观测运行的编排 仿真与策略优化等方面的内容. 这些课题已于近期立项，由 CSST科学工作联合中心管理，正依托北京大学科学中心、 国家天文台科学中心、长三角科学中心和大湾区科学中心 开展研究工作.

巡天观测是CSST在轨运行最重要的一项工作，在其 10年运行的初步规划中, 预计需要累计约7年的时间完成. 同 样地, 巡天数据处理和相应的科学研究工作也是科学数据处 理系统和研究课题的重点. 本文简要介绍CSST及其巡天 计划.

\section{1 巡天空间望远镜}

精确测量引力透镜效应和宇宙大尺度结构的分布，通过 统计分析, 探索暗能量本质、检验引力理论和宇宙学模型是 CSST的驱动性科学目标, 同时也对望远镜的性能和数据处理 的精度提出了严苛的要求. 对于CSST自身, 成像质量是核心, 望远镜口径、观测视场和改变指向的速度是保障巡天效率 的关键.

像质可以用点扩散函数(point spread function, PSF)的属 性来描述. 光学系统的角分辨率在很大程度上反映了其像质, 但分辨率的定义并不唯一，而且对于弱引力透镜效应的精确 测量, PSF的大小和形状都很重要. CSST要求在中心 1.1 平方 度视场内, 包含光学系统和后端模块所有静态误差以及姿态 控制、稳像、微振动等动态因素的PSF $80 \%$ 能量集中度半径 $R_{\mathrm{EE} 80}$ 不大于 $0.15^{\prime \prime}$, PSF椭率不超过 0.15 (表1). 目前设计分析结 果均满足要求. 作为比较, HST高级巡天相机(advanced camera for survey, ACS)中的宽视场相机 (wide field camera, WFC) 在地面使用模拟光学系统测试的结果为 $R_{\mathrm{EE} 80} \sim 0.12$ " (632.8 nm), 在轨使用中心波长在430 833 nm之间的多个滤 光片观测的结果为 $R_{\mathrm{EE} 80} \sim 0.15^{\prime{ }^{[6]}}$. HST与CSST相比, 口径大 了 $20 \%$, PSF中心更为尖锐，具有更高的角分辨率，但其PSF 外围较为延展, 而CSST得益于无遮拦的离轴光学设计, PSF 能量更为集中, 所以其 $R_{\mathrm{EE} 80}$ 指标可以达到与HST相同的水平.

表1中绝对指向精度是指CSST平台使用导星数据标校 星敏感器之后，在没有导星闭环条件下的指向精度，在此基 础上利用导星进行引导，可以显著提高这项指标．对于大视 场巡天，表 1 中的绝对指向精度已经可以很好地满足观测需 求. CSST在约 $400 \mathrm{~km}$ 高的近地轨道上运行, 观测条件变化较 快，为了完成巡天计划，需要在 10 年内进行约 60 万个不同指 向近70万次的观测. 巡天模块观测期间每天拍摄约 300 次 (中 位数), 指向改变的速度对巡天效率有较大影响, 表1中CSST 这项指标已显著优于Euclid、RST等同类项目的相应指标.

表 1 CSST主要技术指标

Table 1 Key specifications of CSST

\begin{tabular}{ccc}
\hline 指标项 & 指标要求 & 备注 \\
\hline 主镜口径 & $2 \mathrm{~m}$ & \\
焦距 & $28 \mathrm{~m}$ & \\
巡天视场面积 & $\geqslant 1.1$ 平方度 & \\
观测波长 & $0.25 ~ 1.7 \mu \mathrm{m}, 590 \sim 730 \mu \mathrm{m}$ & 巡天观测 $0.255 \sim 1.0 \mu \mathrm{m}$ \\
$P S F R_{\mathrm{EE} 80}$ & $\leqslant 0.15^{\prime \prime}$ & $\lambda=632.8 \mathrm{~nm}, 1.1$ 平方度视场内, 含静态像质、 \\
PSF椭率 & 平均 $\leqslant 0.05$, 最大 $\leqslant 0.15$ & $3 \sigma, 300 \mathrm{~s}$ 内, 使用导星 \\
稳像精度 & 指向 $\leqslant 0.05^{\prime \prime}$, 绕光轴 $\leqslant 1.5^{\prime \prime}$ & $3 \sigma$ \\
微振动 & $\leqslant 0.01^{\prime \prime}$ & $3 \sigma$, 使用导星标校 \\
绝对指向精度 & 指向 $\leqslant 5^{\prime \prime}$, 绕光轴 $\leqslant 10^{\prime \prime}$ & $45^{\circ}$ 以上 $0.35^{\circ} / \mathrm{s}$, 含稳定时间 \\
指向改变速度 & $1^{\circ} 50 \mathrm{~s}, 20^{\circ} 100 \mathrm{~s}, 45^{\circ} 150 \mathrm{~s}$ &
\end{tabular}


为了获得大视场、高像质的优异性能, CSST光学系统 采用Cook型离轴三镜消像散光学设计(图2), 同时增加了一 面折转镜, 实现不同终端仪器之间的切换、调焦和精密稳像 的功能. 整个光路中没有遮拦, 镜面支撑也都在光路之外, 不 仅通光效率高，而且更重要的是，PSF能量集中，没有镜面支 撑造成的衍射图案(图3), 有利于弱引力透镜剪切效应的测量. 此外, CSST还采用了主动光学技术, 可在轨调整次镜准直, 进 一步保障像质.

\section{2 巡天模块}

巡天模块是CSST最重要的一台观测设备，主要由巡天 主焦面与天体定标组件、短波红外组件、滤光片组件、无 缝光谱组件、快门组件、主体结构、热控与制冷、巡天主 焦面与天体定标焦面电子学、主控电子学、定标光源等构 成, 其主体的结构分解见图4. 主光机的波前传感器(wavefront sensor, WFS; 用于测量波前误差, 供主动光学矫正用)和精细 导星仪(fine guidance sensor, FGS; 用于确定指向及稳像反馈) 也安装在巡天模块上.

表2列出了巡天模块的主要光电性能指标. 成像波段的
名义起止波长对应滤光片通光曲线半高全宽的特征波长，系 统通光效率在 $1.05 \mu \mathrm{m}$ 以上波长处由探测器的量子效率截止. 无缝光谱观测同样使用滤光片限制带宽，波段起止波长定义 与成像波段的相同，但仅用于规定最小波长范围. 限于模块 包络的约束, 无缝光谱色散元件离焦面不足 $80 \mathrm{~mm}$, 光谱色 散角度大，若采用棱栅，则存在楔角大、元件过厚过重、像 质难以保证等问题，因此采用透射式闪耀光栅在成像光路里 直接色散实现无缝光谱观测.

巡天模块焦面布局如图5所示. 主焦面使用视场中心约 $1.1^{\circ} \times 1.2^{\circ}$ 的区域，由 30 片探测器拼接而成，其中 18 片用于多 波段成像观测(NUV、u、g、r、i、z、y), 12片用于无缝光 谱观测 $(G U 、 G V 、 G I)$. 两侧像质稍差的视场内安置了天体 定标组件(photometric calibration unit, PCU; 用于观测较亮的 标准星)、短波红外组件(near-infraread unit, NIU; 0.9 1.7 $\mu \mathrm{m}$ ) 和主光机的WFS与FGS. 主焦面与天体定标组件使用相同的 探测器, 像元尺寸为 $10 \mu \mathrm{m} \times 10 \mu \mathrm{m}$, 器件规模为 $9 \mathrm{k} \times 9 \mathrm{k}$, 目前 仍在评估国内外若干厂商的产品. 短波红外组件计划使用 8 片 $640 \times 512$ 规模、 $15 \mu \mathrm{m}$ 像元的焦平面器件, 工作于 $80 \mathrm{~K}$ 低 温, 对 $0.9 \sim 1.3$ 和 $1.3 \sim 1.7 \mu \mathrm{m}$ 两个波段进行成像和无缝光谱观
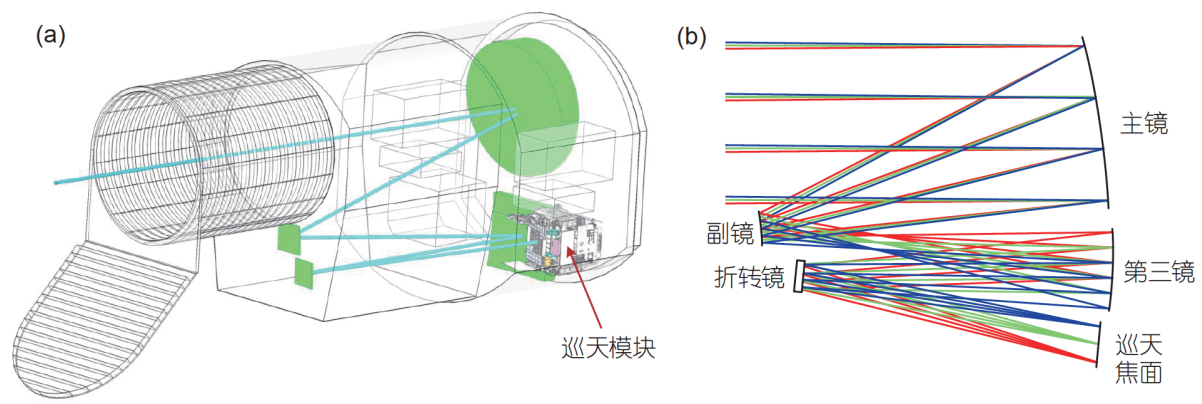

图 2 巡天模块在CSST光学系统内部的位置示意(a)和Cook型离轴三镜消像散光学设计示意(b)

Figure 2 Illustration of the survey camera inside the CSST optical system (a) and Cook-type off-axis three-mirror anastigmatic optical design (b)
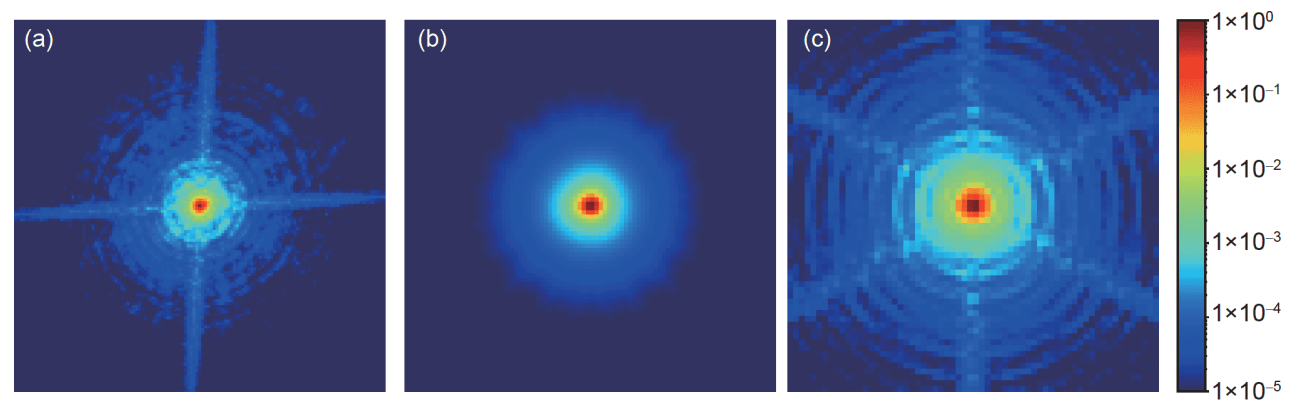

图 3 使用Tiny $\operatorname{Tim}^{[7]}$ 软件计算的HST $\operatorname{PSF}\left((\mathrm{a})\right.$, 像元张角 $\left.0.049^{\prime \prime}\right) 、 \operatorname{CSST}$ 光学设计 $\operatorname{PSF}\left(R_{\mathrm{EE} 80}=0.12^{\prime \prime}\right)$ 退化到 $R_{\mathrm{EE} 80}=0.145^{\prime \prime}$ 的结果 $((\mathrm{b})$, 像元张角 0.074 "; 光学模型由中国科学院长春光学精密机械与物理研究所提供)和参考Euclid光学系统设计得到的PSF((c), 像元张角 $0.010^{\prime \prime}$, PSF数据由中 国科学院国家天文台南京天文光学技术研究所提供), 显示范围均为 $6.4^{\prime \prime} \times 6.4^{\prime \prime}$

Figure 3 HST PSF calculated using Tiny $\operatorname{Tim}^{[7]}$ ((a), pixel size $\left.0.049^{\prime \prime}\right)$, CSST optics designed PSF $\left(R_{\mathrm{EE} 80}=0.12^{\prime \prime}\right)$ degraded to $R_{\mathrm{EE} 80}=0.145^{\prime \prime}((\mathrm{b})$, pixel size 0.074"; optical model is provided by Changchun Institute of Optics, Fine Mechanics and Physics, Chinese Academy of Sciences), and designed PSF of a Euclid-like optical system ((c), pixel size 0.10", PSF data are provided by Nanjing Institute of Astronomical Optics \& Technology, National Astronomical Observatories, Chinese Academy of Sciences), all shown in the same stamp size of $6.4^{\prime \prime} \times 6.4^{\prime \prime}$ 


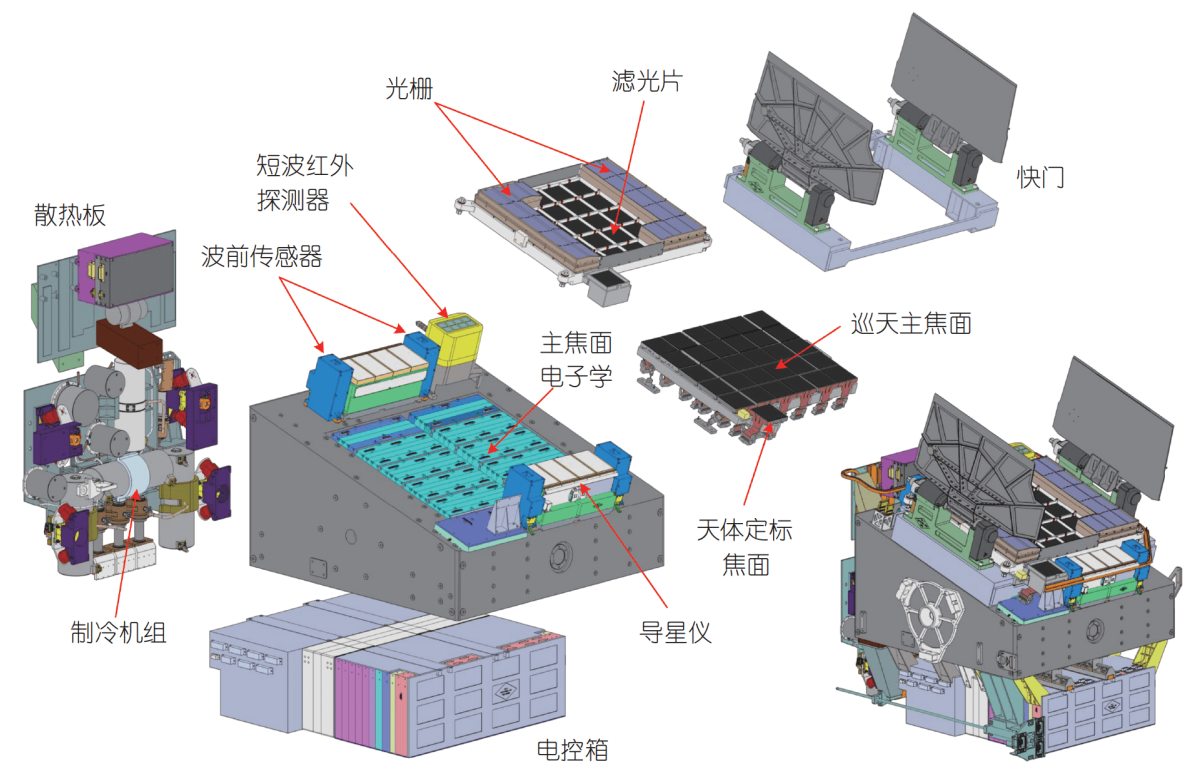

图 4 巡天模块结构分解图

Figure 4 Exploded view of the CSST survey camera

表 2 巡天模块主要光电性能指标

Table 2 Key opto-electrical specifications of the CSST survey camera

\begin{tabular}{|c|c|c|}
\hline 指标项 & 指标要求 & 备注 \\
\hline 主焦面感光面积 & $\geqslant 234000 \mathrm{~mm}^{2}$ & \\
\hline 成像观测波段 & 覆盖0.255 1.0 $\mu \mathrm{m}$, 至少6个波段 & $\begin{array}{l}\text { 设计: NUV } 252 \sim 321 \mathrm{~nm} \text {; u } 321 \sim 401 \mathrm{~nm} \text {; g } 401 \sim 547 \mathrm{~nm} \text {; } \\
\text { r } 547 \sim 692 \mathrm{~nm} \text {; i } 692 \sim 842 \mathrm{~nm} \text {; z } 842 \sim 1080 \mathrm{~nm} \text {; } 927 \sim 1080 \mathrm{~nm}\end{array}$ \\
\hline 光谱观测波段 & 覆盖0.255 1.0 $\mu \mathrm{m}$, 至少3个波段 & 设计: GU $255 \sim 400 \mathrm{~nm}$; GV 400 620 nm; GV 620 1000 nm \\
\hline 读出噪声 & $\leqslant 5 \mathrm{e}^{-} / \mathrm{pix}$ & 读出频率 $150 \mathrm{kHz}$ \\
\hline 暗电流 & $\leqslant 0.02 \mathrm{e}^{-} /($pix s $)$ & 探测器工况温度 $-85^{\circ} \mathrm{C}$ \\
\hline 满阱电荷数 & 最低值 $\geqslant 70 \mathrm{ke}^{-}$, 平均值 $\geqslant 90 \mathrm{ke}^{-}$ & \\
\hline 图像量化位数 & 16位 & \\
\hline 光谱分辨率 & $R \geqslant 200(R=\lambda / \Delta \lambda)$ & 各光栅视场内不同区域和波长的平均值 \\
\hline 成像 $R_{\mathrm{EE} 80}$ & $\begin{array}{c}\mathrm{NUV} / \mathrm{u} / \mathrm{g} / \mathrm{r} \text { 波段 } \leqslant 0.135^{\prime \prime} ; \mathrm{i} \text { 波段 } \leqslant 0.145^{\prime \prime} ; \\
\text { Z波段 } \leqslant 0.165^{\prime \prime}\end{array}$ & $\begin{array}{c}\text { 按每个波段的中心波长考核, 主光机静态像质: NUV/u/g/r波 } \\
\text { 段 } \leqslant 0.13^{\prime \prime} ; \text { i波段 } \leqslant 0.14^{\prime \prime} ; \text { z波段 } \leqslant 0.16^{\prime \prime}\end{array}$ \\
\hline 光谱 $R_{\mathrm{EE} 80}$ & $\begin{array}{c}\text { 所用的 }+1 \text { 或 }-1 \text { 级光谱以及 } 0 \text { 级像 } R_{\mathrm{EE} 80} \text { 均 } \\
\text { 值 } \leqslant 0.3^{\prime \prime}, \text { 最大值 } \leqslant 0.4^{\prime \prime}\end{array}$ & 各光栅视场内的平均值, 按每个波段的中心波长考核 \\
\hline
\end{tabular}

测, 谱分辨率 $R \geqslant 100$, 读出噪声 $\leqslant 50 \quad \mathrm{e}^{-} / \mathrm{pix}$, 暗电流 $\leqslant 5 \mathrm{e}^{-} \mathrm{pix}^{-1} \mathrm{~s}^{-1}$. 该器件由中国科学院上海技术物理研究所研 发. 虽然国产短波红外器件与国外产品性能有较大差距, 但 基于CSST的口径和分辨率，仍可在高红移天体、褐矮星、 太阳系天体、系外行星等研究方向取得有价值的成果.

巡天主焦面规模庞大，尺寸约 $500 \mathrm{~mm} \times 600 \mathrm{~mm}$ (不包括 FGS等周边组件). 模块的包络、重量资源有限, 难以采用滤 光轮的方案切换滤光片和光栅, 而且空间运动机构始终是影 响可靠性和寿命的风险点. 因此, 巡天模块采用每片探测器 固定观测一个波段的方案，不仅可以规避技术风险和困难，
而且每个波段均可选择使用在其带通范围内量子效率最高 的探测器, 从而提升整个系统各波段内的通光效率.

巡天模块无缝光谱仪方案如图6所示. 用于无缝光谱观 测的每片探测器对应 2 片光栅, 即GU、GV和GI每个光栅单 元都由 2 片光栅组成, 这 2 片光栅工作级次 $(+1$ 级或 -1 级 $)$ 的光 谱都向对方的方向色散, 带内衍射效率 $55 \%$ 65\%, 光谱长度 3.4 4.1 mm. 0级像的位置与直接成像位置相同(假设将光栅 替换成材料、厚度和安装倾角相同的滤光片), 无色散, 像质 较好, 其能量占人射总能量 $10 \%$ 的水平, 可作为波长定标的参 考点. 如果每片探测器只使用 1 片光栅色散, 则单片光栅尺寸 


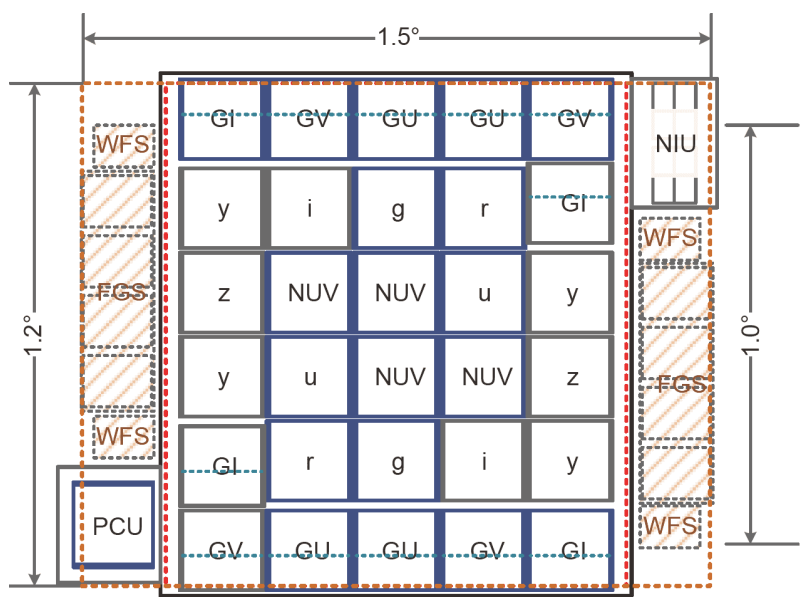

图 5 巡天模块焦面布局

Figure 5 Focal plane arrangement of the CSST survey camera

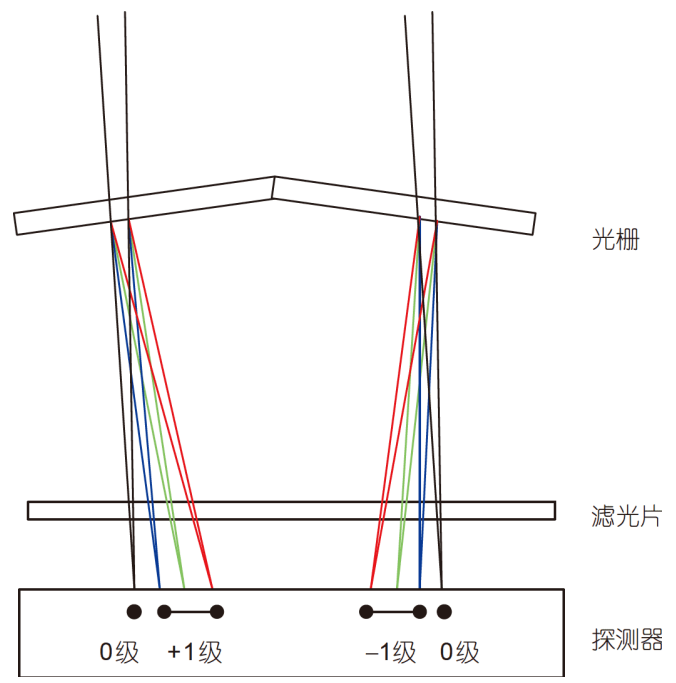

图 6 巡天模块无缝光谱仪方案示意

Figure 6 Schematic of the slitless spectrograph of the CSST survey camera

将近 $100 \mathrm{~mm} \times 100 \mathrm{~mm}$, 在如此大的范围内保证光谱像质满足 要求相当困难, 且由于光栅需要与焦面倾斜安装, 离焦面越近, 光谱分辨率越低，使用单片大尺寸光栅会加剧光谱分辨率随 视场位置变化的问题. 无论使用1或2片光栅, 若探测器范围内 的光谱工作级次均向一个方向色散, 则 0 级像位于该方向探测 器边缘天体的光谱必然落在探测器之外，造成光谱有效视场 的损失. 巡天模块所采用的方案可保证两片光栅的光谱工作 级次均落在探测器上, 避免了光谱有效视场的损失, 但在两片 光栅搭接处, 因结构挡光和衍射仍然会损失一部分视场, 并增 加天体光谱混叠的概率. 对于CSST巡天重点观测的中高银纬 天区，绝大部分情况下无㖓光谱可探测的恒星和星系每平方 角分仅若干个，因两片光栅搭接处工作级次的光谱交叉而造 成的光谱混叠概率很小, 所造成的光谱数据损失可以接受.
图7展示了基于主光机各镜面反射率、巡天模块滤光片 透过率(设计曲线, 含预估的工艺损失)、光栅效率(设计曲线, 含预估的工艺损失)和Teledyne e2v公司CCD290-99标准硅工 艺增强型UV0镀膜(蓝端优化)、标准硅工艺Astro Multi-2镀 膜(可见光宽波段优化)以及深耗尽工艺Astro Multi-2镀膜(红 端优化)的量子效率计算得到的系统通光效率. 作为比较, HST ACS相机在这些波段内的系统通光效率(见Advanced Camera for Surveys Instrument Handbook, 以下简称为AC$\mathrm{SIH})$ 仅为图7中曲线的 $60 \%$ 左右.

\section{3 巡天计划与探测能力}

观测宇宙学研究需要大量的河外天体样本, 所以CSST 的巡天观测以恒星密度和黄道光背景都较低的中高银纬、 中高黄纬天区为主. 目前任务规划中, CSST巡天包括四部分: 17500 平方度多色成像观测、与之同天区的无缝光谱观测、 400 平方度的多色成像深场观测和与之同天区的无缝光谱深 场观测, 主要参数见表 3 . 其中, 极限星等的计算采用了HST平 均黄道光背景的水平(见ACSIH). 比 400 平方度深场深 1 个星 等以上的 9 平方度深场观测、低银纬天区观测、微引力透镜 观测以及太阳系天体的观测计划正在论证中.

巡天模块的探测器与滤光片和光栅固定安装, 观测编排 中以每个探测器为单元覆盖整个天区. 主焦面任意一片探测 器完成一次天区覆盖, 那么该天区除边界之外的区域也被所 有其他探测器完整覆盖一次. 每个波段重复观测该天区的次 数取决于该波段分配的探测器的数量(图5). 观测天区的边界 不能被所有波段覆盖，或部分波段重复观测次数少于天区内 部该波段的重复观测次数，面积越小的天区，这样的边界区 域占比越大, 所以巡天任务中每个深场的面积不宜太小.

由于地球和太阳对近地轨道观测条件的影响, CSST观 测方向的序列较为随机，难以保证各波段有序地覆盖每一个 方向，也难以设定每个方向、每个波段的重复观测时间间隔. 因此, 如果按常规的时域研究项目的要求来考量, CSST巡天

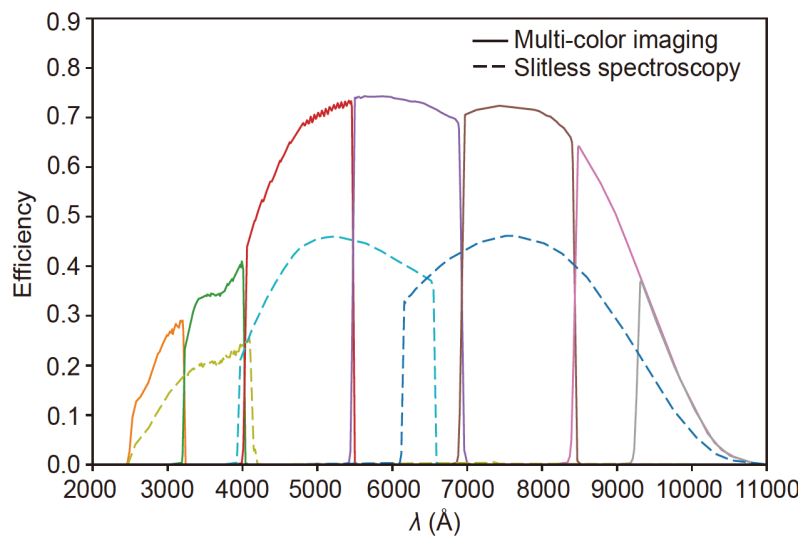

图 7 CSST巡天观测各波段系统通光效率

Figure 7 System throughput of CSST survey bands 
表 3 CSST巡天主要参数

Table 3 Key parameters of the CSST survey

\begin{tabular}{|c|c|c|c|c|c|c|c|c|c|}
\hline 观测手段 & $\begin{array}{l}\text { 天区面积 } \\
\text { (平方度) }\end{array}$ & 曝光时间(s) & & & & 源, 5 & & & \\
\hline \multirow{4}{*}{ 多色成像 } & & & $\mathrm{NUV}^{\mathrm{a})}$ & $\mathrm{u}$ & $\mathrm{g}$ & $\mathrm{r}$ & i & $\mathrm{z}$ & $y^{a)}$ \\
\hline & 17500 & $150 \times 2$ & 25.4 & 25.4 & 26.3 & 26.0 & 25.9 & 25.2 & 24.4 \\
\hline & 400 & $250 \times 8$ & 26.7 & 26.7 & 27.5 & 27.2 & 27.0 & 26.4 & 25.7 \\
\hline & & & & GU & & GV & & GI & \\
\hline \multirow{2}{*}{ 无缝光谱 } & 17500 & $150 \times 4$ & & 23.2 & & 23.4 & & 23.2 & \\
\hline & 400 & $250 \times 16$ & & 24.4 & & 24.5 & & 24.3 & \\
\hline
\end{tabular}

a) NUV和y波段曝光次数与无缝光谱波段的曝光次数相同

缺乏优势, 但是CSST必然会观测到大量的变源、暂现源和移 动源. 如何利用CSST巡天数据, 或者将CSST与其他巡天项目 相结合, 识别和研究这些天体, 值得深人地探讨.

巡天编排策略对观测运行进度有很大的影响, 目前正在 针对观测需求进行优化. 图8给出了17500平方度多色成像观 测运行进度的一组仿真结果. 图中被任意一片滤光片观测过 的天区面积(红色曲线)在运行初期增长很快, 1 年内即可覆盖 约 12000 平方度, 这个面积在中后期增长缓慢, 因为中后期主 要是在补全每个方向上的观测波段. 图中被所有滤光片按规 定次数完成观测的天区面积(绿色曲线)则是在初期增长缓 慢, 中后期增长速度有显著提高.

CSST在轨运行 10 年期间与空间站有 4 次计划中的停靠, 对应图 8 中各曲线上 4 个天区面积不增长的时间段. 巡天模块 在图8的结果中累计使用了CSST $64 \%$ 的运行时间. 现有的观 测编排仿真主要针对累计运行时间进行了优化, 尚未深人考 虑各个研究方向特定的观测需求和专门的定标观测时间以 及故障、维修等因素, 因此, 为了确保 10 年内能够完成所规

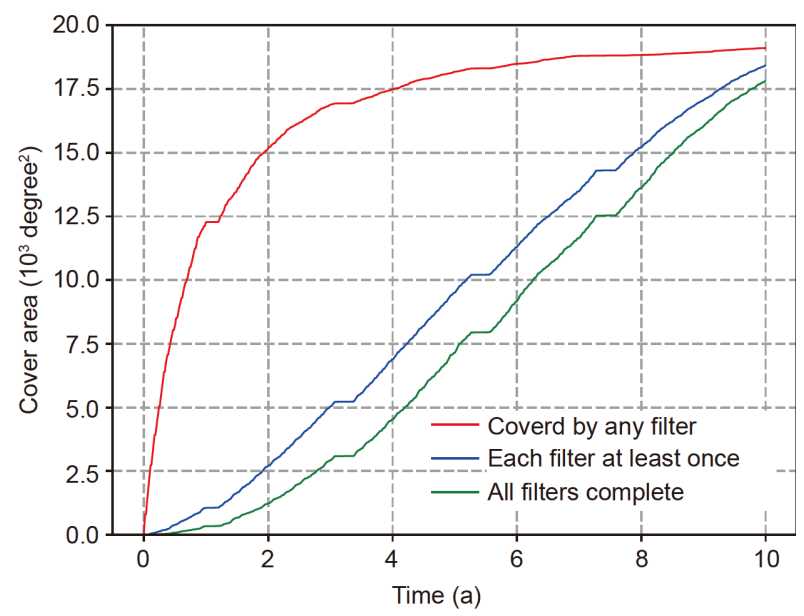

图 8 CSST巡天大面积多色成像观测运行进度仿真结果

Figure 8 Progress of the CSST large-area multiband imaging survey in one realization of operations simulation
划的巡天, 计划分配 $70 \%$ 的运行时间用于巡天观测。

基于星系测光样本的研究往往需要使用测光数据估计 星系的红移. 图9比较了两组按不同信噪比阈值选取的CSST 星系模拟样本的测光红移精度. 为了获得更真实的效果, 首 先以 1.7 平方度的HST $\operatorname{COSMOS}^{[8]}$ 多次曝光叠加处理后的科 学图像为模板, 使用其多波段测光星表 ${ }^{[9]}$, 借助拟合出的星 系能谱分布，按CSST各波段的光电性能、天光背景、成像 和像元分辨率生成模拟观测图像. 然后, 从模拟观测图像上 提取星系，进行测光，剔除少量与原星表无法交叉匹配的星 系, 形成模拟样本. 最后, 按 $\mathrm{g}$ 或 $\mathrm{r}$ 波段信噪比 $\mathrm{SNR}_{\mathrm{g} \text { or } \mathrm{i}} \geqslant 10$ (图9 (a)) 和 $\mathrm{SNR}_{\mathrm{g}}$ or $\mathrm{i} \geqslant 20($ 图9(b))选取两个子样本, 用LéPhare软 件 ${ }^{[10,11]}$ 计算测光红移.

图9中蓝色虚线标出了 $\mid z_{\mathrm{fit}}-z_{\text {input }} /\left(1+z_{\text {input }}\right) \leqslant 0.15$ 的区域, 此范围外的数据点计为离群点, 不计人测光红移弥散 (标准 差)的计算中 ${ }^{[11]}$. 可以看到, $\mathrm{SNR}_{\mathrm{g}}$ or $\mathrm{i} \geqslant 20$ 样本相比 $\mathrm{SNR}_{\mathrm{g} \text { or } \mathrm{i}} \geqslant 10$ 样本, 测光红移弥散从 0.030 减少到 0.023 , 离群 点比例从 $7.4 \%$ 降至 $2.4 \%$, 改善显著, 但同时星系面密度减少 了一半以上. 降低测光红移误差有助于提升基于星系多色成 像数据大样本统计的各种宇宙学探针的探测能力 ${ }^{[12,13]}$, 而用 于剪切(场)测量的星系的面密度则是弱引力透镜效应统计的 重要误差源, 需要尽可能提高. 考虑到测光红移比剪切测量 对星系样本选择的限制更强 ${ }^{[14]}$, 实际工作的重点在于提高低 信噪比样本中星系的测光红移精度. 例如, 将CSST的NUV、 $u 、 g 、 r 、 i 、 z 、 y$ 测光数据与Euclid的 $Y 、 J 、 H$ 测光数据相 结合, 即可显著提高测光红移精度 ${ }^{[15]}$. 此外, 在CSST宇宙学 数据分析工作中也将充分利用自身样本内部、各个宇宙学 探针之间以及自身样本与外部光谱红移深场样本之间的交 叉关联, 对样本的测光红移和其他系统误差进行(自)校准, 加 强精确宇宙学方向的研究能力 ${ }^{[12,16-18]}$.

\section{4 总结与展望}

天文探测能力的不断提升推动着人类对宇宙认知的进 步，每一次观测深度、广度、波段、测光精度、空间分辨 

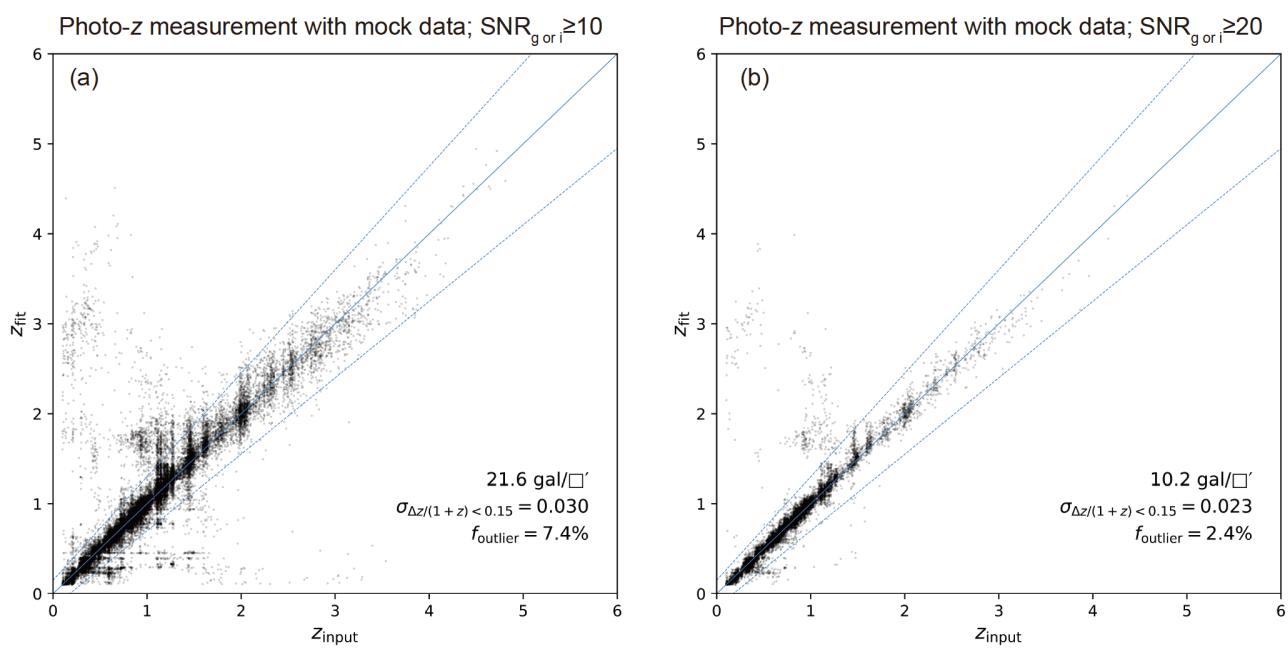

图 9 CSST星系模拟样本测光红移与输人红移比较. (a) $\mathrm{SNR}_{\mathrm{g} \text { or } \mathrm{i}} \geqslant 10$; (b) $\mathrm{SNR}_{\mathrm{g} \text { or } \mathrm{i}} \geqslant 20$

Figure 9 Comparison of photometric redshifts and input redshifts of the CSST galaxy mock sample. (a) $\mathrm{SNR}_{\mathrm{g} \text { or } \mathrm{i}} \geqslant 10$; (b) $\mathrm{SNR}_{\mathrm{g} \text { or } \mathrm{i}} \geqslant 20$

\section{表 4 国际同期巡天项目比较}

Table 4 Comparison of contemporary survey projects

\begin{tabular}{|c|c|c|c|c|c|c|c|c|c|c|}
\hline 项目 & 轨道/台址 & 口径(m) & 科学运行 & $\begin{array}{c}\text { 视场 } \\
\text { (平方度) }\end{array}$ & $R_{\mathrm{EE} 80}(")$ & $\begin{array}{c}\text { 像元数量 } \\
\text { (亿) }\end{array}$ & $\begin{array}{l}\text { 巡天面积 } \\
\text { (平方度) }\end{array}$ & $\begin{array}{c}\text { 巡天观测波 } \\
\text { 长(nm) }\end{array}$ & 成像波段数 & 光谱波段数 \\
\hline CSST & 近地轨道 & 2 & 2024年前后 & 1.1 & 0.15 & $31^{\text {a) }}$ & 17500 & $255 \sim 1000$ & 7 & 3 \\
\hline Euclid & 日地L2点 & 1.2 & 计划2022年 & $\begin{array}{l}0.56 \\
0.55\end{array}$ & $\begin{array}{l}0.23 \\
0.63\end{array}$ & $\begin{array}{l}6.1 \\
0.7\end{array}$ & 15000 & $\begin{array}{c}550 \sim 900 \\
920 \sim 2000\end{array}$ & $\begin{array}{l}1 \\
3\end{array}$ & $\begin{array}{c}\text { 无 } \\
1\end{array}$ \\
\hline RST & 日地L2点 & 2.4 & 不早于2025年 & 0.28 & 0.24 & 3 & 2000 & 927 2000 & 4 & 1 \\
\hline VRO & 智利 & 8.4 & 2022年 & 9.6 & 0.54 & 32 & 18000 & $320 \sim 1050$ & 6 & 无 \\
\hline
\end{tabular}

a) 巡天主焦面约 25 亿像元, 导星仪约6亿像元

率、谱分辨率以及时间分辨率等方面的突破，都会带来重大 的发现, 甚至引发天文学和物理学革命性的发展. CSST大规 模多色成像与无缝光谱巡天的指标在上述多个方面不仅大 幅超越以往项目, 而且在同期巡天项目中像质 $\left(R_{\mathrm{EE} 80}\right)$ 最好, 近 紫外波段的观测能力独一无二(表4), 综合性能优异，具有很 强的竞争力. 同时, CSST的科学任务不只是巡天, 其配备的 5 台第一代观测终端都极具特色, 或是仪器性能指标, 或是观 测任务, 都致力在前沿研究方向上实现突破.

从多个天文项目的成功经验来看，一方面，科学目标对 项目的实施起着驱动性的关键作用，但另一方面，最终的科 学成果往往远超出原先所能想象的范畴, 这是由科学研究的 探索性所决定的. 从一定意义上说, 这些项目最为成功之处 在于打开了新的发现空间，不断激发新的思想，不断取得新 的认识. 以曾经安装在托洛洛山美洲际天文台(Cerro Tololo
Inter-American Observatory) $4 \mathrm{~m}$ 望远镜(后命名为Blanco望远 镜)上的Big Throughput Camera ${ }^{[19]}$ (BTC)为例, 1996年开放使 用时, 是当时唯一一台实际运行的背照式CCD拼接焦面天文 相机，BTC这个名称也反映了其技术指标在当时的先进性 BTC与Blanco望远镜配合，可以在一周内完成数平方度深至 $r \sim 26$ 等的观测. 两个团队使用BTC搜寻I $I$ 型超新星, 试图测量 宇宙膨胀的“减速因子”，但他们的发现跟预期恰恰相反一 宇宙的膨胀正在加速 ${ }^{[20,21]}$.

揭示宇宙加速膨胀的机理正是多个下一代大型巡天项 目的驱动性科学目标. 可以预期, CSST将与欧美同期的 VRO、Euclid、RST等标志性项目并驾齐驱，并在优势方向 上有所超越，不仅在宇宙加速膨胀机理的研究方面有望取得 突破，而且将打开更广阔的发现空间，为天文学的发展作出 重要贡献. 
的前期论证和部分技术攻关工作得到了国家自然科学基金(11033005)、中国科学院战略性先导科技专项空间科学预先 研究项目(XDA04070800)、中国科学院天文台站设备更新及重大仪器设备运行专项、中国科学院事业单位修缮购置 专项、中国科学院空间科学研究院培育项目、国家天文台预研项目和天文联合基金(U1931208)资助.中国空间技术研 究院, 中国科学院长春光学精密机械与物理研究所、国家天文台、光电技术研究所、上海技术物理研究所、西安光学 精密机械研究所和国家天文台南京天文光学技术研究所的承研团队为本文提供了素材, 国家天文台孟宪民、伏西洋、 许优华和柏勇制作了部分图件, 在此向他们表示感谢.

\section{推荐阅读文献}

1 York D G, Adelman J, Anderson J E, et al. The sloan digital sky survey: Technical summary. Astron J, 2000, 120: 1579-1587

2 Gao M, Zhao G H, Gu Y D. Space science and application mission in China's space station (in Chinese). Bull Chin Acad Sci, 2015, 30: 721-732 [高铭, 赵光恒, 顾逸东. 我国空间站的空间科学与应用任务. 中国科学院院刊, 2015, 30: 721-732]

3 Zhan H. Consideration for a large-scale multi-color imaging and slitless spectroscopy survey on the Chinese space station and its application in dark energy research (in Chinese). Sci Sin-Phys Mech Astron, 2011, 41: 1441-1447 [詹虎. 中国空间站大规模多色测光与无缝光谱巡天的设想 及其在暗能量研究领域的应用. 中国科学: 物理学 力学 天文学, 2011, 41: 1441-1447]

4 Zhan H. In: 42nd COSPAR Scientific Assembly (Vancouver: COSPAR), 2018, E1.16-4-18

5 Su D Q, Cui X Q. Two suggested configurations for the Chinese space telescope. Res Astron Astrophys, 2014, 14: 1055-1060

6 Bohlin R C. Perfecting the photometric calibration of the ACS CCD cameras. Astron J, 2016, 152: 60

7 Krist J E, Hook R N, Stoehr F. Hubble Space Telescope optical modeling using Tiny Tim. Proc SPIE, 2011, 8127: 81270J

8 Scoville N, Aussel H, Brusa M, et al. The Cosmic Evolution Survey (COSMOS): Overview. Astrophys J Suppl Ser, 2007, 172: 1-8

9 Ilbert O, Capak P, Salvato M, et al. Cosmos photometric redshifts with 30-bands for 2-deg ${ }^{2}$. Astrophys J, 2009, 690: 1236-1249

10 Arnouts S, Cristiani S, Moscardini L, et al. Measuring and modelling the redshift evolution of clustering: The Hubble deep field north. Mon Not Roy Astron Soc, 1999, 310: 540-556

11 Ilbert $\mathrm{O}$, Arnouts $\mathrm{S}$, McCracken H J, et al. Accurate photometric redshifts for the CFHT legacy survey calibrated using the VIMOS VLT deep survey. Astron Astrophys, 2006, 457: 841-856

12 Zhan H. Cosmic tomographies: Baryon acoustic oscillations and weak lensing. J Cosmol Astropart Phys, 2006, (8): 008

13 Sun L, Fan Z H, Tao C, et al. Catastrophic photo-z errors and the dark energy parameter estimates with cosmic shear. Astrophys J, 2009, 699: 958967

14 Leauthaud A, Massey R, Kneib J, et al. Weak gravitational lensing with COSMOS: Galaxy selection and shape measurements. Astrophys J Suppl Ser, 2007, 172: 219-238

15 Cao Y, Gong Y, Meng X M, et al. Testing photometric redshift measurements with filter definition of the Chinese Space Station Optical Survey (CSS-OS). Mon Not Roy Astron Soc, 2018, 480: 2178-2190

16 Zhang P, Pen U L, Bernstein G. Self-calibration of photometric redshift scatter in weak-lensing surveys. Mon Not Roy Astron Soc, 2010, 405: 359374

17 Matthews D J, Newman J A. Improving correlation function fitting with ridge regression: Application to crosscorrelation reconstruction. Astrophys J, 2012, 745: 180

18 Zhan H, Tyson J A. Cosmology with the Large Synoptic Survey Telescope: An overview. Rep Prog Phys, 2018, 81: 066901

19 Wittman D M, Tyson J A, Bernstein G M, et al. Big throughput camera: The first year optical astronomical instrumentation. Proc SPIE, 1998, 3355: 626-634

20 Riess A G, Filippenko A V, Challis P, et al. Observational evidence from supernovae for an accelerating Universe and a cosmological constant. Astron J, 1998, 116: 1009-1038

21 Perlmutter S, Aldering G, Goldhaber G, et al. Measurements of $\Omega$ and $\Lambda$ from 42 high-redshift supernovae. Astrophys J, 1999, 517: 565-586 


\title{
The wide-field multiband imaging and slitless spectroscopy survey to be carried out by the Survey Space Telescope of China Manned Space Program
}

\author{
$\mathrm{Hu}$ Zhan $^{1,2}$ \\ ${ }^{1}$ National Astronomical Observatories, Chinese Academy of Sciences, Beijing 100101, China; \\ ${ }^{2}$ Kavli Institute for Astronomy and Astrophysics, Peking University, Beijing 100871, China \\ E-mail: zhanhu@nao.cas.cn
}

The 2 m-aperture Survey Space Telescope (also known as the China Space Station Telescope, CSST) is a major science project of China Manned Space Program. It is expected to start science operations around 2024 and has a nominal mission lifetime of 10 years. During observations, CSST will fly independently in the same orbit as the space station while maintaining a large distance apart. It can dock with the space station for refueling and servicing as scheduled or as needed. With a Cook-type three-mirror anastigmat design, CSST can achieve superior image quality within a large field of view (FoV), which gives it an advantage for survey observations. Being an off-axis telescope without any obstruction, its point spread function (PSF) is not affected by diffraction of mirror support structures and is thus helpful for weak-lensing shear measurements. The radius encircling $80 \%$ energy of the PSF within CSST's central 1.1 square degrees of FoV is required to be no more than $0.15^{\prime \prime}$, including all wavefront errors in the optics and instruments and dynamical effects such as the telescope's attitude control and vibration.

CSST will be launched with 5 first-generation instruments including a survey camera, a terahertz receiver, a multichannel imager, an integral field spectrograph, and a cool planet imaging coronagraph. The survey camera is equipped with 30 $9 \mathrm{k} \times 9 \mathrm{k}$ detectors for science observations, each with a filter or grating mounted above, a defocused $9 \mathrm{k} \times 9 \mathrm{k}$ detector for flux calibration in $\mathrm{r}$ band, $8640 \times 512$ near-infrared detectors, fine guide sensors, and wavefront sensors. The primary task of CSST is to carry out a high-resolution large-area multiband imaging and slitless spectroscopy survey covering the wavelength range of 255-1000 $\mathrm{nm}$. Precision cosmology is the main science driver of the survey, and, therefore, the main area of sky selected for observation is at median-to-high galactic latitude and median-to-high ecliptic latitude. It will take the survey camera roughly 7 years of operation accumulated over 10 years of orbital time to image roughly 17500 square degrees of the sky in NUV, u, g, r, i, z, and y bands and take slitless spectroscopy of the same sky in 3 bands. The pointsource $5 \sigma$ limiting magnitudes in $\mathrm{g}$ and $\mathrm{r}$ bands can reach 26 (AB mag) or higher. The spectral resolution $(R=\lambda / \Delta \lambda)$ of the slitless spectrograph is specified to be on average no less than 200, and the wide-band-equivalent limiting magnitudes in GV $(400-620 \mathrm{~nm})$ and GI $(620-1000 \mathrm{~nm})$ bands will reach 23 or higher. In addition, a number of deep fields will be selected for more observations to reach at least one magnitude deeper than the wide-area survey.

The combined advantage of its angular resolution, depth, wavelength range, capability of both imaging and spectroscopy and large-area sky coverage makes the CSST survey highly competitive, and, at the same time, its observations are also highly complementary with other large projects of its time, such as Vera Rubin Observatory, Euclid, and Roman Space Telescope. These projects will provide extremely rich datasets for potential breakthroughs in studies from the solar system to cosmology and beyond.

space telescope, wide-field survey, slitless spectroscopy, cosmology, photometric redshift doi: 10.1360/TB-2021-0016 